the late-onset asthma group (OR 1.28 [95\% CI: 1.11-1.48]) or the early-onset persistent asthma group (OR 1.27 [95\% CI: 1.08-1.48]) compared to the never/infrequent asthma group. Similar results were seen for boys, OR 1.16 [95\% CI: $1.08-$ 1.25], OR 1.11 [95\% CI: 0.98-1.25], and OR 1.34 [95\% CI: $1.20-1.51$ ] respectively. The odds remained largely the same in imputed and unadjusted models.

Conclusion In a Danish nationwide population four asthma trajectories were identified, in agreement with clinical studies. ACE in early life was associated with all asthma phenotypes for both boys and girls.

\section{P.2.24 THE EFFECT OF THE EXPOSURE TO WORK-RELATED POTENTIALLY TRAUMATIC EVENTS ON OCCUPATIONAL FUNCTIONING (WORK LIMITATIONS) AMONG FIREFIGHTERS: A CROSS-SECTIONAL STUDY}

Ki Soo Park*. Gyeongsang National University, Jinju, South Korea

\subsection{6/OEM-2019-EPI.256}

Objective The purpose of this study was to investigate whether firefighters' exposure to work-related trauma events is related to work limitations that form part of social health.

Methods In 2016, 618 firefighters from four cities in Gyeongsangnam-do participated in this study. They were analyzed using a health-related work limitations questionnaire, experience and type of work-related trauma, Korea Depression Scale, and the World Health Organization quality of life assessment instrument. The health-related work limitations consisted of three scales: 'physical work demands,' 'psychosocial work demands,' and 'environmental work demands.' The analysis was done by hierarchical logistic regression analysis.

Results Of the subjects, $70.2 \%$ remembered traumatic experiences they had experienced during their job. The total work limitations were $21.5 \%$; the limitation of physical work demands was $16.8 \%$, that of psychosocial work demands was $15.5 \%$, and that of environmental work demands was $13.8 \%$. Logistic regression analysis of the final model 3 showed that, if firefighters have experience of their own risks and personal injury, physical (95\% CI=1.169-9.405), psychosocial (95\% CI $=1.080-8.609$ ), and environmental (95\% CI=1.65914.402), the total $(95 \% \mathrm{CI}=1.439-9.678)$ work limitation was significantly increased. When there was a memory of a terrible body or injury, the total work limitations increased significantly (95\% CI=1.092-3.905).

Conclusions When firefighters are exposed to trauma events during their jobs, these have a negative impact on their work. In order to protect and improve the occupational functions of firefighters, effective prevention and management of workrelated trauma incidents should be considered.

\section{P.2.27 MANGANESE EXPOSURE IN STEEL AND ALLIED FACTORY: CAUSE OF SECONDARY PARKINSONISM}

${ }^{1}$ Priyanka Roy*, ${ }^{2}$ Praveen Kumar. 'Department of Labour, Kolkata, India; ${ }^{2}$ Consultant Neurologist, Durgapur, India

\subsection{6/OEM-2019-EPI.257}

Case presentation A 43 year old male presented with 6 months history of gradual onset and progressive bradykinesia, tremors of upper limbs and walking difficulty in the form of imbalance and short shuffling gait, slurring of speech with hypophonia. Neurological examination revealed slow broken saccades, rigidity with cogwheeling, bradykinesia and short shuffling, festinant gait. He had action and postural tremors of upper limbs. Pull test was positive. Thus a diagnosis of young onset Parkinsonism was considered. But, occupational history revealed that he worked in blast furnace of a steel and allied factory for past 14 years where manganese ore has been using for the strengthening of the stainless steel. He was evaluated with MRI Brain which showed symmetrical hyperintensities involving basal ganglia and subcortical white matter in T2 weighted images. T1 weighted images showed evidence of basal ganglia hyperintensities probably related to mineral deposition. Renal function, thyroid function tests including anti thyroid antibodies, KF Ring, S. Ceruloplasmin, Liver function tests, CSF, ultrasound abdomen- all were normal. Two consecutive serum manganese level tests in six months interval showed initial rise (two times) with the history of exposure and followed by normal level without the exposure. Patient was started on symptomatic medications like levodopa and trihexiphenidyl but after 8 months of medication (March- October, 2018) he had not showed any significant improvement. The written consent was taken from patient for this study.

Conclusion In view of strong occupational history of manganese exposure, the clinical features and non responsiveness of treatment with levodopa, a possibility of manganese toxicity was strongly considered for this rare case of secondary parkinsonism. Also non adherence with the legal mandate of periodical medical examination of workers exposed to toxic metals with long term consequence is very important factor to be corrected in the developing country like India.

\section{P.2.29 DOES A CHANGE IN WORKING CONDITIONS INFLUENCE LABOUR FORCE PARTICIPATION AMONG WORKERS WITH A CHRONIC DISEASE?}

${ }^{1}$ Jolinda Schram*, 'Suzan Robroek, ${ }^{2}$ Patricia Ots, ${ }^{2}$ Sander van Zon, ${ }^{2}$ Sandra Brouwer, ${ }^{1}$ Alex Burdorf, ${ }^{1,3}$ Karen Oude Hengel. 'Erasmus Mc Rotterdam, Department of Public Health, Rotterdam, Netherlands; 'University Medical Center Groningen, Department of Health Sciences, Community and Occupational Medicine, Groningen, Netherlands; ${ }^{3}$ Netherlands Organization for Applied Scientific Research TNO, Leiden, Netherlands

\subsection{6/OEM-2019-EPI.258}

Objective This study investigated the association between changing working conditions and exit from paid employment during the following year among older workers with a chronic disease in the Netherlands.

Method Four annual waves from the Study on Transitions in Employment, Ability and Motivation (STREAM; 2010-2013) provided information on working conditions and demographics for 2838 older workers with a chronic disease, aged 45-64 years. The analytical sample consisted of 5491 responses from 2838 workers. Five types of working conditions were investigated; physical workload, psychological job demands, job autonomy, emotional job demands and social support. Discrete-time survival models were used to estimate the associations of change in working conditions in a particular year on the probability of exiting paid work for persons with a chronic disease in the following year.

Results Of the 2838 workers, a small majority was male $(52 \%)$, most workers had an intermediate level of education (39.7\%), and the mean age was 53.7 years (SD 5.50). Results 